\title{
Phenomenology of hydromagnetic turbulence in a uniformly expanding medium
}

\author{
WILLIAM H. MATTHAEUS, \\ GARY P. ZANK ${ }^{1}$ and SEAN OUGHTON \\ ${ }^{1}$ Bartol Research Institute, University of Delaware, Newark, Delaware 19716, USA \\ ${ }^{2}$ Department of Mathematics, University College London, London WClE 6BT, UK
}

(Received 22 May 1996)

A simple phenomenology is developed for the decay and transport of turbulence in a constant-speed, uniformly expanding medium. The fluctuations are assumed to be locally incompressible, and either of the hydrodynamic or non-Alfvénic magnetohydrodynamic (MHD) type. In order to represent local effects of nonlinearities, a simple model of the Kármán-Dryden type for locally homogeneous turbulent decay is adopted. A detailed discussion of the parameters of this familiar one-point hydrodynamic closure is given, which has been shown recently to be applicable to non-Alfvénic MHD as well. The effects of the large-scale flow and expansion are incorporated using a two-scale approach, in which assumptions of particular turbulence symmetries provide simplifications. The derived model is tractable and provides a basis for understanding turbulence in the outer heliosphere, as well as in other astrophysical applications.

\section{Introduction}

While the theory of turbulence is of great fundamental interest, in many theoretical applications there is a need to represent the effects of turbulence in a simple and reasonably accurate way within the confines of a broader physics model. The motivation for the present paper is a familiar situation of this type that occurs in space and astrophysical plasmas: a wind or other radially expanding flow carries with it evolving plasma turbulence. A prime example, and the main application that we have in mind, is the solar wind (Barnes 1979; Goldstein et al. 1995). However, similar ideas may be relevant to stellar winds, and to the turbulent expansion of supernova remnants and galactic winds.

Our goal is to develop a model that will provide a basic understanding of plasma turbulence in these expanding media, adopting a magnetohydrodynamic (MHD) approximation. The most basic information sought is the radial profile of both the turbulent energy and the local decay rates, for turbulence that is either freely decaying during its radial transport, or else driven in a way that is readily modelled. For simplicity, we shall assume that the large-scale flow is spherically symmetric with a constant speed which is everywhere greater than the Alfven speed associated with the large-scale magnetic field. The fluctuations are assumed to be short-wavelength, so that we can exploit scale-separation to develop a transport theory. This suggests 
the treatment of nonlinearities as locally homogeneous, an approximation that has some observational support in the solar wind (Matthaeus and Goldstein 1982a,b).

We shall adopt as basic variables the relevant turbulent energy densities (per unit mass), and associated outer length scales, that characterize the size of interacting energy-containing eddies. This minimal representation allows connections to be made with observed or inferred radial (or temporal) profiles of turbulent fluctuations. Another issue that influences the degree of complexity of this model is the rotational symmetry of the fluctuations. If no assumptions are made, the transport theory involves the full correlation tensors, and transport of individual turbulent components (Marsch and Tu 1989; Zhou and Matthaeus 1990a). However, if a fixed symmetry is assumed to be maintained, there is considerable simplification. Here we shall assume either isotropic fluctuations or fluctuations that are two-dimensional (2D) relative to a specified axis. The $2 \mathrm{D}$ model is motivated by recent observational studies of the solar wind (Matthaeus et al. 1990; Bieber et al. 1996).

To ensure a tractable model, which might nonetheless be relevant to many circumstances, we adopt the simplifying approximation that the MHD fluctuations have zero cross-helicity, meaning that the magnetic and velocity field fluctuations are uncorrelated. This type of turbulence is often referred to as non-Alfvénic. In addition, we assume that the ratio of kinetic energy to magnetic energy in the fluctuations is independent of time and uniform in space. Observed conditions in the outer heliosphere (Roberts et al. 1987a,b) are also consistent with these approximations.

The output of the model is the space and time dependence of the fluctuation energies and the correlation length scale(s). The model also provides decay rates of the turbulence. These in principle are connected with the radial profile of the turbulent heating rate, although we shall not pursue this here.

The models we obtain are related in obvious ways to ' $K-\epsilon$ ' engineering models of hydrodynamic turbulence (Jones and Launder 1972; Bradshaw et al. 1981). Much of the main content of the present models can be extracted, through various assumptions, from the much more complex MHD transport models of Zhou and Matthaeus (1990a), Tu and Marsch $(1990,1993)$ and Matthaeus et al. (1994). Indeed, the daunting algebraic complexity of these full MHD spectral transport models is an important motivation for the present effort to extract physically intuitive simplifications of the expanding small-scale turbulence problem. Connections to these theories will be pointed out at various points in the presentation.

As a first step, in the following section we describe a scale-separated transport theory for locally homogeneous hydrodynamic turbulence. In Sec. 3 we discuss a class of familiar simple phenomenologies for homogeneous hydrodynamic turbulence. We emphasize how the choice of parameters in these models corresponds to distinet physical assumptions within the context of the original assumptions. Sections 4 and 5 assemble the MHD scale-separated transport model for zero cross helicity and constant ratio of kinetic to magnetic energy densities. In Sec. 6, both general and special solutions are presented and discussed.

\section{Hydrodynamic turbulence in a uniform radial wind}

Let the fluid velocity field $\mathbf{V}=\mathbf{U}+\mathbf{u}$ be separated into a mean flow $\mathbf{U}$ and a fluctuating part $\mathbf{u}$. For an appropriate space-time (or ensemble) averaging procedure $\langle\cdots\rangle$, let us define $\mathbf{U} \equiv\langle\mathbf{V}\rangle$, and require $\langle\mathbf{u}\rangle=0$. The mean flow $\mathbf{U}$ varies only 
slowly in space, and the rapid spatial variations of the fluctuation $\mathbf{u}$ forces it to vanish under the averaging procedure. In the usual way, we start from the NavierStokes equation $\rho(\partial \mathbf{V} / \partial t+\mathbf{V} \cdot \nabla \mathbf{V})=-\nabla p+\mathbf{d}$, with pressure $p$ and dissipative term $d$. We assume that the fluctuations are short-wavelength compared with the scale of variation of the mean field $\mathbf{U}$, and that the small-scale fluctuations are incompressible, $\boldsymbol{\nabla} \cdot \mathbf{u}=\mathbf{0}$.

An equation for the evolution of the two point correlation tensor $R_{i j}(\mathbf{r})=\left\langle u_{i} u_{j}^{\prime}\right\rangle \equiv$ $\left\langle u_{i}(\mathbf{x}) u_{j}(\mathbf{x}+\mathbf{r})\right\rangle$ (the prime' denotes separation by spatial lag $\mathbf{r}$ ) follows by a standard procedure: The mean of the Navier-Stokes equation is subtracted from the full equation, providing an equation for the evolution of $\mathbf{u}$. The $i$ th component of this equation is multiplied by $u_{j}^{\prime}$ and ensemble-averaged; the trace of the result is

$$
\frac{\partial R_{i i}(\mathbf{r})}{\partial t}+\mathbf{U} \cdot \nabla R_{i i}(\mathbf{r})+2 R_{i j}^{s}(\mathbf{r}) \nabla_{j} U_{i}=N L,
$$

where $R_{i j}^{s}=R_{i j}+R_{j i}$, the nonlinear, dissipative and pressure terms are grouped into the right-hand side and designated by $N L$, and the summation convention on repeated indices is observed. The transport equation for the mean square fluctuation amplitude (twice the fluctuation energy per unit mass) follows by evaluating the previous equation at $\mathbf{r}=0$ :

$$
\frac{\partial u^{2}}{\partial t}+\mathbf{U} \cdot \nabla u^{2}+2\left\langle u_{i} u_{j}\right\rangle \nabla_{j} U_{i}=N L_{u}
$$

To evaluate basic models of the nonlinear term $N L_{u}$, a dynamically evolving length scale is required. A convenient choice is the correlation length $\lambda_{w}=L_{w} / u^{2}$, defined in terms of the correlation integral $L_{w}$. The direction $w$ is chosen temporarily to be the $\hat{\mathbf{1}}$ direction, in a locally Cartesian right-handed coordinate system with axes in the $\hat{\mathbf{1}}, \hat{\mathbf{2}}$, $\hat{\mathbf{z}}$ directions, so that

$$
L_{w}=\int_{0}^{\infty} d w R_{i i}(w, 0,0)
$$

Below we shall make specific choices for the $w$ direction. The time development of $L_{w}$ follows by integrating (2.1) over $x$ with the other components of $\mathbf{r}$ held at 0 . Thus we obtain

$$
\frac{\partial L_{w}}{\partial t}+\mathrm{U} \cdot \nabla L_{w}+2\left[\int_{0}^{\infty} R_{i j}^{s}(w, 0,0) d w\right] \nabla_{i} U_{j}=N L_{L}
$$

where now $N L_{L}$ denotes the effects of associated nonlinear terms upon $L_{x}$.

The tensor contraction terms on the right hand sides of Eqs. (2.2) and (2.4) depend on the geometry of both the fluctuations and the large-scale flow. For the present case of interest, uniform radial expansion at speed $U$, relative to a local Cartesian coordinate frame with radial direction $\hat{\mathbf{r}}=\hat{\mathbf{1}}$, we find

$$
\nabla_{i} U_{j}=\left(\begin{array}{lll}
0 & 0 & 0 \\
0 & 1 & 0 \\
0 & 0 & 1
\end{array}\right) \frac{U}{r}
$$

These terms simplify further under the assumption that the turbulence has fixed rotational symmetry properties, independent of the radial coordinate. Here we consider two possible choices: isotropic turbulence, for which $\left\langle u_{i} u_{j}\right\rangle=\frac{1}{3} \delta_{i j} u^{2}$, and locally two-dimensional (2D) turbulence that is isotropic in a two-dimensional plane 
perpendicular to a direction designated by $\mathbf{B}_{0}$ that lies at an angle $\Psi$ relative to the outward radial direction $\hat{\mathbf{r}}$.

For isotropic turbulence and uniform outflow, $\left\langle u_{i} u_{j}\right\rangle \nabla_{j} U_{i}=\frac{2}{3} u^{2} U / r$. It is straightforward to show that

$$
2\left[\int_{0}^{\infty} R_{i j}^{s}(w) d w\right] \nabla_{i} U_{j}=\frac{B_{u 3} L_{w} U}{r}
$$

where the constant parameter $B_{u 3}$, specific to the $3 \mathrm{D}$ isotropic hydrodynamic case, depends upon the choice of the direction of the coordinate $w$, the correlation function having zero argument for the two directions transverse to $w$. If the correlation length is measured in the $r$ direction, $L_{w} \rightarrow L_{r}$ and $B_{u 3}=1$; if the correlation length is measured in a direction normal to the radial, $L_{w} \rightarrow L_{N}$ and $B_{u 3}=\frac{3}{2}$

For the 2D case, the variance matrix, in a coordinate system in which $\mathbf{B}_{0}=B_{0} \hat{\mathbf{1}}$, is just $\frac{\mathbf{l}}{2}(\mathbf{I}-\hat{\mathbf{1}} \mathbf{\mathbf { 1 }}) u^{2}$; rotating this by an angle $\Psi$ brings the radial to the $\hat{\mathbf{1}}$ direction. After then contracting with (2.5), we find for this case that

$$
2\left\langle u_{i} u_{j}\right\rangle \nabla_{j} U_{i}=U_{r}^{2}\left(1+\cos ^{2} \Psi\right)
$$

To compute the correlation integral for $2 \mathrm{D}$ turbulence, again starting from a $\hat{\mathbf{B}}_{0}=\hat{\mathbf{1}}$ coordinate system, we adopt the convention that the correlation integral is computed by integrating in the $\hat{\mathbf{2}}$ direction, but the relationship of this coordinate system to the radial direction is not yet established. For this coordinate system, it is easy to see that

$$
\int_{0}^{\infty}\left\{R_{i j}^{2 D}(0, y, 0)\right\} d y=\left(\begin{array}{ccc}
0 & 0 & 0 \\
0 & L_{2} & 0 \\
0 & 0 & 0
\end{array}\right)
$$

where $L_{2}=\int R_{\alpha \alpha}(0, y, 0) d y$. Upon rotating by angle $\Psi$ so that $\mathbf{r}$ coincides with the $\hat{\mathbf{1}}$ direction, we find that

$$
2\left[\int_{0}^{\infty} R_{i j}^{2 D}(w) d w\right] \nabla_{i} U_{j}=B_{u 2} \frac{L_{2} U}{r} .
$$

The parameter $B_{u 2}$, for the 2D case, has the value $B_{u 2}=2 \cos ^{2} \Psi$ when the correlation integral is in the $\left(r, B_{0}\right)$ plane, and has the value $B_{u 2}=2$ when the correlation direction $w$ is normal to both the $r$ and $B_{0}$ directions.

The isotropic and $2 \mathrm{D}$ results can be summarized by the pair of equations

$$
\begin{aligned}
& \frac{\partial u^{2}}{\partial t}+U \frac{\partial u^{2}}{\partial r}+\frac{A U}{r} u^{2}=N L_{u}, \\
& \partial L_{w}+U \frac{\partial L_{w}}{\partial r}+\frac{B U}{r} L_{w}=N L_{L} .
\end{aligned}
$$

The values of the parameters $A$ and $B$ corresponding to the several cases discussed above are summarized in Table 1 . In order to complete the model, we must now establish explicit models for the nonlinear terms.

\section{Local phenomenology and constants}

In this section we ignore effects of spatial inhomogeneity and discuss the simplest class of phenomenologies for homogeneous turbulence. The model decay equations 
Table 1. Values of the parameters $A$ and $B$ for use in (2.10), (2.11), (5.4), and (4.12). Values of $C$ for (5.7) are given by $C=B-A$. Parameters are given for several hydrodynamic (HD) and MHD models described in the text, including isotropic and 2D models. Two closures are included for $L_{D}$. Appropriate parameters are given for correlation lengths that are radial $\left(L_{r}\right)$, in the $\left(r, B_{0}\right)$ plane $\left(L_{r B}\right)$ for $2 D$ fluctuations, or normal to the radial $\left(L_{N}\right)$. Also shown is the asymptotic $r \rightarrow \infty$ behaviour of the fluctuation energy (per unit mass) and the correlation scale (using either $L_{r}$ or $L_{r}$ ).

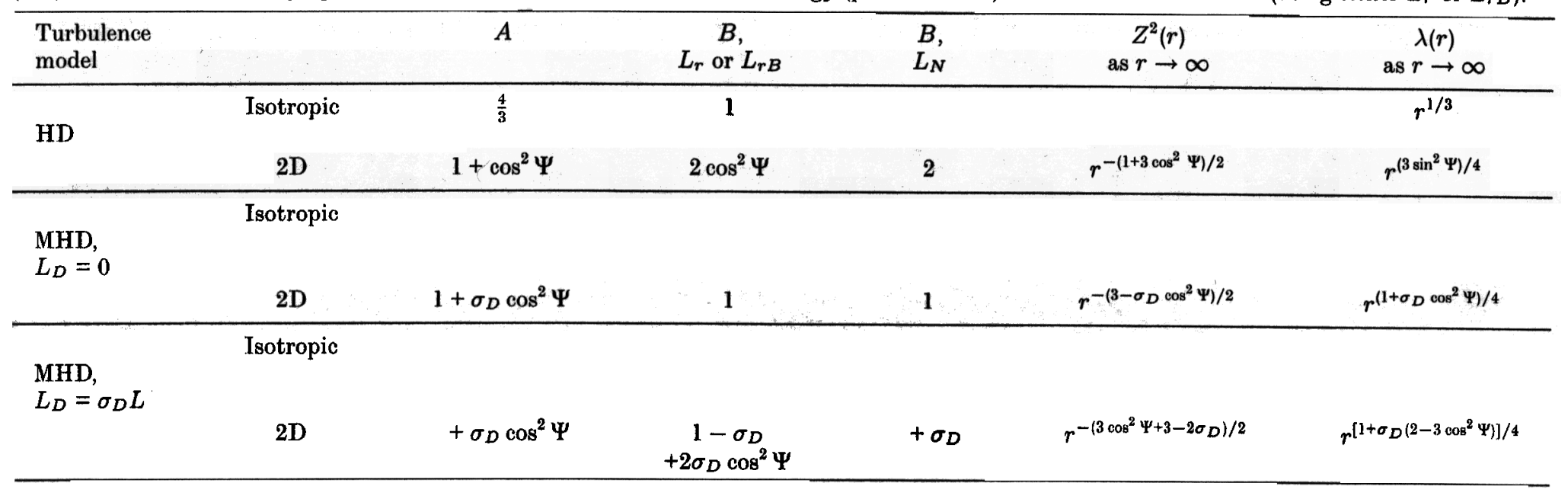


for the RMS velocity $u(t)$ and a characteristic (large-scale) length $\ell(t)$ (with both quantities assumed to have the same characteristic decay time $\tau(t)=\ell / u$ ) are

$$
\begin{aligned}
\frac{d u^{2}}{d t} & =-\alpha \frac{u^{3}}{\ell}, \\
\frac{d \ell}{d t} & =\beta u,
\end{aligned}
$$

with $\alpha, \beta \geqslant 0$. We refer to them as the Kármán-Dryden equations. These equations are implicit in Kármán and Howarth (1938), but it seems Dryden (1943) was the first to write them down explicitly (see his p. 15, where his $A \equiv \frac{1}{2} \alpha$ and $B \equiv \beta$ ). Taylor's (1935) model is equivalent to (3.1) with $\beta=0$ (i.e. $\ell=$ constant).

One frequently chooses $\ell$ to be the longitudinal correlation length $\lambda_{\|}$, with $\lambda_{\|} u^{2} \propto$ $\int_{0}^{\infty} R_{11}(x, 0,0) d x$. However, we make the association introduced in the previous section - that $\ell=\lambda_{w}$, with the direction $w$ determined according to the problem at hand. For isotropic turbulence, $\lambda_{w}=2 \lambda_{\|}$; for $2 \mathrm{D}$ turbulence with $w$ perpendicular to the ignorable direction, $\lambda_{w}=\lambda_{\|}$.

Equations (3.1) and (3.2) have exact solutions, first discussed by Dryden (1943). Observing that $u^{2} \ell^{\alpha / \beta}=$ const yields (Hossain et al. 1995)

$$
\begin{aligned}
& u(t)=u\left(t_{0}\right)\left[1+A\left(t-t_{0}\right)\right]^{-\alpha /(\alpha+2 \beta)}, \\
& \ell(t)=\ell\left(t_{0}\right)\left[1+A\left(t-t_{0}\right)\right]^{2 \beta /(\alpha+2 \beta)},
\end{aligned}
$$

where the characteristic time for the development of self-similar decay is

$$
\frac{1}{A}=\underset{\alpha+2 \beta}{2 \beta} \frac{l_{0}}{u_{0}},
$$

and the zero subscript signifies evaluation at $t=t_{0}$. Note that $A$ depends on the absolute values of $\alpha$ and $\beta$.

For $t \gg 1 / A$, these are power-law solutions, depending only on the ratio $\alpha / 2 \beta$. Experiments suggest that, approximately, $u^{2}(t) \propto 1 / t$, implying that $\alpha / 2 \beta=1$ or $\alpha=2 \beta$, i.e. a straight line through the origin in the $(\alpha, \beta)$ plane, as depicted in Fig. 1.

If we require that there really is only one time scale in the decay, i.e. $\tau=A^{-1}$, we must have

$$
\alpha=2-2 \beta,
$$

another straight line in the $(\alpha, \beta)$ plane.

Thus, if we insist on $(a)$ a single time scale, and $(b)$ matching the 'experimental decay law' then this mandates $\alpha=1$ and $\beta=\frac{1}{2}$. Orszag (1977) provides another argument for why $\ell \sim t^{1 / 2}$ and $u^{2} \sim 1 / t$, which relies on perfect (not just asymptotic) power-law forms of the solutions, and clearly connects these values of the constants with the Kármán-Howarth (1938) hypothesis of self-preservation of the shape of the correlation functions during decay. In brief, Orszag argues as follows, for isotropic turbulence.

(a) It is exactly true that

$$
\frac{d u^{2}}{d t} \propto \int_{0}^{\infty} k^{2} E(k, t) d k \propto \frac{u^{2}}{\lambda_{T}^{2}}
$$




\section{Phenomenology of turbulence in an expanding medium}

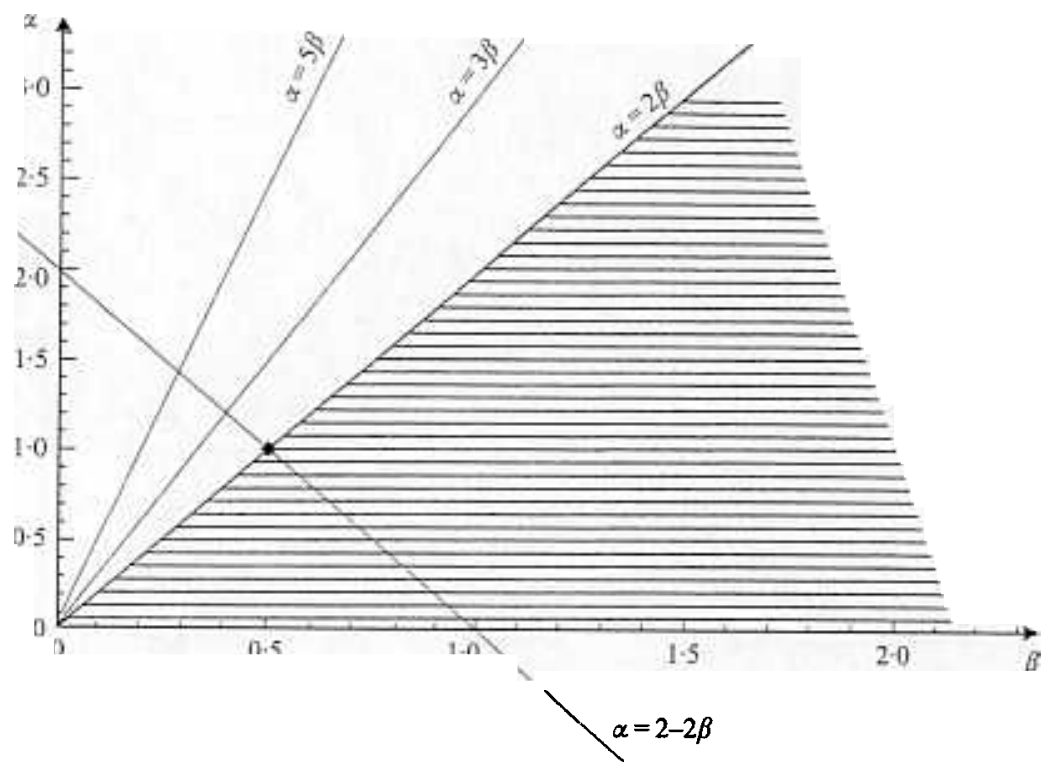

Figure 1. Schematic diagram of the values of $\alpha$ and $\beta$ that form the parameter space for the decay model, (3.1) and (3.2). The lines indicate values appropriate for constant Reynolds number $(\alpha=2 \beta)$, Saffman's model $(\alpha=3 \beta)$, Kolmogorov's model $(\alpha=5 \beta)$ and the values for which the two characteristic time scales are equal $(\alpha=2-2 \beta)$. The point corresponding to the Kármán-Howarth self-preservation property is $\alpha=1, \beta=\frac{1}{2}$, indicted by the small black box. Shaded values are disallowed because the Reynolds number is increasing in time.

where $E(k, t)$ is the omnidirectional energy spectrum and $\lambda_{T}$ is the Taylor microscale.

(b) Suppose that the spectrum of the decaying turbulence is of a self-preserving form at all scales:

$$
E(k)=u^{2}(t) \ell(t) F(k \ell(t))
$$

with $\int_{0}^{\infty} F(x) d x=1$.

(c) Substituting this into (3.7) yields

$$
\frac{d u^{2}}{d t} \propto \frac{u^{2}}{\ell^{2}} \int_{0}^{\infty} x^{2} F(x) d x
$$

(d) Assuming a power law for energy decay, i.e. $u^{2}(t) \propto\left(t-t_{0}\right)^{-n},(3.9)$ implies that $\ell \propto\left(t-t_{0}\right)^{1 / 2}$. In fact, any length scale characterizing the turbulence could be used in place of $\ell$, since they are all proportional to each other by virtue of the self-preservation assumption (3.8).

(e) Assuming further that (3.1) holds, it follows that $n=1$. Comparing with the exact solutions (3.3) and (3.4) of the model decay equations (3.1) and (3.2), the correspondence of $\alpha=1$ and $\beta=\frac{1}{2}$ to the Kármán-Howarth assumption of self-preservation is evident.

Dryden (1943) and later Kármán and Lin $(1949,1987)$ showed that self-similar decay for all scales requires, in addition, constancy of the Reynolds number $u \ell / \nu$, 
or equivalently that

$$
\frac{d}{d t}(u \ell)=0
$$

since the kinematic viscosity, $\nu$ is assumed constant (and uniform)

Now, from (3.1) and (3.2),

$$
\frac{d}{d t}(u \ell)=\left(\beta-\frac{1}{2} \alpha\right) u^{2}
$$

Clearly, the case of self-similar decay corresponds to $\alpha=2 \beta$, the same curve required for matching with experimental results at 'late' times. Furthermore, since it is unphysical for the Reynolds number to increase with time in unforced, decaying flows, we must have

$$
\alpha \geqslant 2 \beta \text {. }
$$

Thus the portion of the $(\alpha, \beta)$ plane below the line $\alpha=2 \beta$ is not relevant (and is shaded in Fig. 1). Note that the quantity $u \ell$ can also be thought of as a turbulent viscosity, and the (physically) allowed region of parameter space in Fig. 1 is that for which the turbulent viscosity is non-increasing, while values $\alpha=1$ and $\beta=\frac{1}{2}$ correspond to a constant turbulent viscosity.

There are also various theories of turbulent decay that involve departures from self-preservation. A number of these can also be cast into the simple form of (3.1) and (3.2), including the models of Comte-Bellot and Corrsin $(1966 ; \alpha=\beta(r+1)$ for $r=$ const), Kolmogorov (1941; $\alpha=5 \beta$ ), Saffman $(1967 ; \alpha=3 \beta$ ) and Taylor (1935; $\beta=0$, i.e. $\ell=$ const). These models also yield straight lines in the $(\alpha, \beta)$ plane (Fig. 1). The intersections of these lines with the single timescale line (3.6), provides perhaps the best way to determine the appropriate absolute values of $\alpha$ and $\beta$. Each of these theories is based upon specific physical properties that may be of relevance to the choice of constants. For example, Kolmogorov's (1941) model is based upon the observation that invariance of the Loitsianskii integral requires a departure from self-preservation at the longest scales. The family of models considered by Comte-Bellot and Corrsin (1966) makes an assumption of a non-self-preserving power-law energy spectrum at the very longest scales, and includes Kolmogorov's model as a special case. Taylor's (1935) original assumption that $\ell=$ const gives energy behaving as $u^{2}(t) \sim t^{-2}$, and may be most appropriate when 'finite-box-size' effects limit the growth of the correlation scale.

So far, direct simulations show that at best $\alpha$ and $\beta$ only tend asymptotically to constants (see e.g. Hossain et al. 1995); it is to be hoped that this is only because attainable Reynolds numbers are too small. However, Hossain et al. (1995) find that $\alpha=1, \beta=\frac{1}{2}$ is a reasonable choice of parameters for 3D periodic hydrodynamic turbulence at large-scale Reynolds numbers of a few hundred.

\section{MHD turbulence in a spherical wind}

MHD turbulence involves a magnetic field $\mathbf{B}$ that can be expressed as a mean plus a fluctuating component, i.e. $\mathbf{B}=\mathbf{B}_{0}+\mathbf{b}$, where $\langle\mathbf{B}\rangle=\mathbf{B}_{0}$ is slowly varying in space, and the angle bracket is defined as in the hydrodynamic case. It is convenient to discuss the phenomenology of MHD decay in terms of the Elsässer (1950) representation 


\section{Phenomenology of turbulence in an expanding medium}

by defining

$$
\mathbf{z}^{ \pm} \equiv \mathbf{v} \pm \frac{\mathbf{b}}{(4 \pi \rho)^{1 / 2}}=\mathbf{v} \pm \mathbf{v}_{A}
$$

where $\mathbf{v}$ is the velocity, $b$ is the magnetic field and $\nabla_{A}$ is the magnetic field in Alfvén speed units. The plasma density $\rho$ is assumed constant and $\mathbf{V}_{A}=\mathbf{B}_{0} /(4 \pi \rho)^{1 / 2}$ denotes the large-scale magnetic field in Alfvén speed units.

Of particular relevance here are estimates for the turbulent decay of the meansquare values of the Elsässer fields, $Z_{+}^{2} \equiv\left\langle\left|\mathbf{z}^{+}\right|^{2}\right\rangle$ and $Z_{-}^{2} \equiv\left\langle\left|\mathbf{z}^{-}\right|^{2}\right\rangle$, and of the mean value $D \equiv\left\langle\mathbf{z}^{+} \cdot \mathbf{z}^{-}\right\rangle$, where $\langle\cdots\rangle$ denotes a volume average over a representative parcel of homogeneous turbulence. The kinetic and magnetic energies per unit mass of the fluctuations are $E_{v}=\frac{1}{2}\left\langle|\mathbf{v}|^{2}\right\rangle \equiv \frac{1}{2} u^{2}$ and $E_{b}=\frac{1}{2}\left\langle\left|\nabla_{A}\right|^{2}\right\rangle \equiv \frac{1}{2} v_{A}^{2}$, and the total turbulent energy per unit mass is $E=E_{v}+E_{b}$. Here we shall assume the special case of vanishing cross-helicity, $H_{c}=\frac{1}{2}\left\langle v \cdot \nabla_{A}\right\rangle=0$; this type of fluctuation is often called 'non-Alfvenic' because the fluctuations lack the correlations between velocity and magnetic field that are characteristic of unidirectionally propagating largeamplitude Alfvén waves such as those observed in the inner solar wind (Belcher and Davis 1971). The difference between the kinetic and magnetic energies (i.e. the residual energy) is $E_{D} \equiv E_{v}-E_{b}=\frac{1}{2} D$, and is assumed here to have a value that is a specified fraction of the fluctuation energy density. That is, we specify

$$
\sigma_{D}=\frac{D}{2 E}=\frac{E_{v}-E_{b}}{E_{v}+E_{b}}
$$

We associate correlation lengths $\lambda_{+}$and $\lambda_{-}$with the $Z_{+}^{2}$ and $Z_{-}^{2}$ fluctuations, respectively, and a correlation length $\lambda_{D}$ with the energy difference $D$. These lengths reflect the typical size of eddies in the energy-containing range. In analogy to the hydrodynamic definitions, the integrals $L_{ \pm}=\int H_{i i}^{ \pm} d w$ over the correlation functions $H_{i j}=\left\langle z_{i}^{ \pm} z_{j}^{ \pm \prime}\right\rangle$ serve to define the correlation lengths $\lambda_{ \pm}$through $L_{ \pm}=Z_{ \pm}^{2} \lambda_{ \pm}$. Similarly, $L_{D}=\int R_{i i}^{D s}(w) d w=D \lambda_{D}$ defines the energy-difference correlation length, where $R_{i j}^{D s}$ is the symmetric part of the cross-correlation $\left\langle z_{i}^{+} z_{j}^{-\prime}\right\rangle$. It is understood that the correlation integrals extend from zero to infinite separation, and will depend in general upon the choice of rectilinear coordinate $w$ in the integration.

The derivation of scale-separated MHD turbulence transport equations for weakly inhomogeneous plasmas proceeds along the lines of the hydrodynamic case, and in particular for the solar wind has been written explicitly by Marsch and Tu (1989), Zhou and Matthaeus (1990a) and Matthaeus et al. (1994). A de novo derivation would start from the compressible MHD equations, and develop transport equations for the correlation functions. The latter, along with auxiliary definitions are given by Zhou and Matthaeus (1990a) as their equations (49)-(52). The equations for the correlation functions $H_{i j}^{ \pm}$couple to several other transport equations, including those for the correlation functions $\left\langle z_{i}^{+} z_{j}^{-\prime}\right\rangle$. The complexity of the full transport formalism is not needed here in view of the several simplifying assumptions we adopt. A significant level of simplification is seen in the transport equations for the energy-containing eddies, given by Matthaeus et al. (1994). However, even that case is more general than what we require here.

A useful starting point is equation (19) of Matthaeus et al. (1994), providing equations for the transport of the Elsassër fluctuation variances $Z_{ \pm}^{2}$. Neglecting terms of order $V_{A} / U$, and noting that we have assumed $Z_{+}^{2}=Z_{-}^{2} \equiv Z^{2}$, these 
reduce to a single equation:

$$
\frac{\partial Z^{2}}{\partial t}+\mathbf{U} \cdot \nabla Z^{2}+Z^{2} \nabla \cdot\left(\frac{1}{2} \mathbf{U}\right)+M D=N_{Z}
$$

The term $N_{Z}$ on the right-hand side represents the nonlinear interactions that are responsible for spectral transfer. The modelling of these terms will be discussed in the next section. The symbol $M$ is the zero-cross-helicity version of the mixing terms $M_{ \pm}$associated with the two Elsässer fields (Zhou and Matthaeus 1990a), and depends upon the assumed symmetry of the small-scale turbulence. In particular, it depends on the form of the symmetric part of the energy-difference correlation tensor $R_{i j}^{D s}(r=0)$ (cf. Zhou and Matthaeus 1990a). If the turbulence is threedimensional then the tensor is simply a multiple of the unit tensor I, while for 2D turbulence in the plane perpendicular to $\mathbf{B}$, it is proportional to $\mathbf{I}-\hat{\mathbf{B}} \hat{\mathbf{B}}$. The explicit forms for zero $H_{c}$ are

$$
\begin{gathered}
M_{3 D}=\nabla \cdot\left(\frac{1}{6} \mathbf{U}\right)=\frac{U}{3 r}, \\
M_{2 D}=\nabla \cdot\left(\frac{1}{2} \mathbf{U}\right)-\hat{\mathbf{B}} \hat{\mathbf{B}}:(\nabla \mathbf{U})=\frac{U \cos ^{2} \Psi}{r} .
\end{gathered}
$$

In the rightmost equalities of (4.4) and (4.5) we have substituted the form of $M$ appropriate for uniform-speed spherical expansion.

The term $M D$ in (4.3) can now be written explicitly for the situations of interest. Making use of (4.2) and the properties of the uniform expansion, we arrive at

$$
(M D)_{3 D}=\frac{U}{3 r} \sigma_{D} Z^{2}
$$

for the $3 \mathrm{D}$ isotropic case, and

$$
(M D)_{2 D}=\cos ^{2} \Psi \frac{U}{r} \sigma_{D} Z^{2}
$$

for the 2D geometry.

As with the hydrodynamic case, an equation will be needed for the correlation scales. Actually, there are two correlation lengths $\lambda_{ \pm}$within the context of the assumptions so far, and it is necessary to make an additional assumption at this time to arrive at a single correlation-length equation. Adopting the condition that $L_{+}=L_{-}$is equivalent to asserting that the areas under the correlation functions $H_{i i}^{+}$ and $H_{i i}^{-}$are equal. Then, since we already have $Z_{+}^{2}=Z_{-}^{2}$, it follows that $\lambda_{+}=\lambda_{-}$. Equation (25) of Matthaeus et al. (1994) then reduces to a single equation for the correlation integral $L=L_{+}=L_{-}$,

$$
\frac{\partial L}{\partial t}+\mathbf{U} \cdot \nabla L+L \nabla \cdot\left(\frac{1}{2} \mathbf{U}\right)+\int \Pi_{i i}(w) d w=N_{L}
$$

where once again terms of order $V_{A} / U$ are omitted. The term involving $\Pi_{i i}$ expresses couplings of the small-scale correlations to the large-scale gradient tensors, and is analogous to the integral term in (2.4). Again there is some freedom in reasonable choices of the direction of integration $\hat{w}$. For $3 \mathrm{D}$ turbulence the choice is somewhat arbitrary, while for $2 \mathrm{D}$ turbulence it is reasonable to restrict it to be orthogonal to $\mathbf{B}_{0}$. In the zero-cross-helicity case $\Pi_{i j}=\Pi_{i j}^{+}=\Pi_{i j}^{-}$, the latter is defined by equation (26) of Matthaeus et al. (1994). In the small- $V_{A} / U$ limit, these 
reduce to

$$
\Pi_{i i}(w)=-\nabla \cdot\left(\frac{1}{2} \mathbf{U}\right) R_{i i}^{D s}(w)+2 \frac{\partial U_{i}}{\partial x_{j}} R_{i j}^{D s}(w)
$$

Integration is over the local, small-scale separation variable $w$, so large-scale variables and their derivatives are treated as constant. There is a constraint among the components of the symmetric part of any solenoidal tensor (Orszag 1977), which $R_{i j}^{D s}$ satisfies for locally incompressible flow, which leads to the convenient expressions

$$
\int_{0}^{\infty} R_{i j}^{D s} d w=L_{D} \frac{\delta_{i j}+\hat{w}_{i} \hat{w}_{j}}{4}
$$

for fully $3 \mathrm{D}$ isotropic turbulence, and

$$
\int_{0}^{\infty} R_{i j}^{D s} d w=L_{D} \hat{w}_{i} \hat{w}_{j}
$$

for $2 \mathrm{D}$ turbulence distributed uniformly normal to the mean magnetic field. The integration is carried out along the unit vector $\hat{\mathbf{w}}$, with $\hat{\mathbf{w}} \cdot \mathbf{B}_{0}=0$ in the $2 \mathrm{D}$ case.

When these expressions are incorporated above, the evolution of $L$ is explicitly coupled to the evolution of $L_{D}$ and to the mean flow. Involvement of $L_{D}$ requires either including an additional model equation for its evolution or making a closure approximation that allows elimination of $L_{D}$. One possibility is to assume that $\lambda_{D}=\lambda$, so that $L_{D}=D \lambda_{D}$ becomes $L_{D}=\sigma_{D} Z^{2} \lambda=\sigma_{D} L$, using (4.2).

For isotropic fluctuations having $R_{i j}^{D s}=\frac{1}{3} \delta_{i j} D$, two possibilities for the direction of computing the integral over $\Pi$ in (4.8) are, once again, the radial $\hat{w}=\hat{\mathbf{r}}$ and the normal $\hat{\mathbf{w}} \cdot \hat{\mathbf{r}}=0$. For the radial- $\hat{\mathbf{w}}$ case, employing (4.10) and (2.5), we conclude that $\int \Pi_{i i}(w) d w=0$. If a direction normal to radial is chosen, we find for the isotropic case that $\int \Pi_{i i}(w) d w=L_{D} U / 2 r$.

For the $2 \mathrm{D}$ case, we let $L_{w} \rightarrow L_{N}$, selecting $\hat{\mathrm{w}}$ to be normal to both the radial and the $\mathbf{B}_{0}$ directions. Using (4.11) gives the result that $\int \Pi_{i i}(w) d w=L_{D} U / r$. For $\hat{\mathrm{w}}$ in the $\left(R, B_{0}\right)$ plane, we designate the correlation integral as $L_{r B}$. In this case the $2 \mathrm{D}$ result becomes $\int \Pi_{i i}(w) d w=\left(2 \cos ^{2} \Psi-1\right) L_{D} U / r$. For each of the above cases, we can use the $L_{D}=\sigma_{D} L$ closure to represent the (4.8) entirely in terms of $L$, at the expense of including the parameter $\sigma_{D}$.

The above approach to the $L_{D}$ closure issue produces a tractable theory, but it suffers from the somewhat arbitrary selection of the correlation direction $\hat{\mathbf{w}}$. Another approach, and one that we have favoured in applications so far of the present ideas, stems from the observation that $L_{D}$, not itself a positive-definite quantity, is actually the difference (Zank et al. 1996) between the area under the velocity field autocorrelation and the area under the magnetic field autocorrelation. If we assert that these areas are equal, it implies that either $\lambda_{D}=0$ or that $D=0$. Since the latter will not in general be enforced, we may adopt the assumption that $\lambda_{D}=0$. This implies then, for all the symmetries of interest, and independent of the choice of $\hat{\mathbf{w}}$, that $\int \Pi_{i i}(w) d w=0$. This eliminates the 'mixing' term, and the appearance of $\sigma_{D}$, in the correlation integral transport equation, and considerably simplifies the transport model. The $L_{D}=0$ approximation appears to be rather reasonable and nearly in accord with the solar wind measurements reported by Matthaeus and Goldstein (1982a).

The results for the MHD correlation integral transport equation are 
summarized by

$$
\frac{\partial L}{\partial t}+U \frac{\partial L}{\partial r}+\frac{B U}{r} L=N_{L}
$$

The coefficient $B$ includes contributions from the last two terms on the left-hand side of (4.8), and depends as well upon the approximation discussed above. The values of $B$ are summarized in Table 1 .

\section{Nonlinear MHD and the transport model}

The remaining task in assembling the MHD phenomenology is to model the locally homogeneous incompressible MHD turbulence effects embodied in the nonlinear terms represented by $N_{Z}$ in (4.3) and by $N_{L}$ in (4.8). The choice of one-point closure models for MHD is, in principle, more complicated than for hydrodynamics for a number of reasons, e.g. effects associated with cross-helicity (Dobrowolny $e t$ al. 1980), Alfvénic propagation effects caused by nonzero $B_{0}$ (Kraichnan 1965), as well as possible effects of the partitioning of energy between kinetic and magnetic ingredients (effects of $D$ ). In the present models, however, $H_{c}=0$, and finite$H_{c}$ effects are not a concern. In addition, several recent computational studies of homogeneous MHD phenomenologies (Hossain et al. 1995; Verma et al. 1996) provide valuable information.

In the spirit of the one-point energy decay model used above for hydrodynamics, we adopt for MHD the model suggested by Hossain et al. (1995), which for homogeneous MHD turbulence has the energy decay equations

$$
\frac{d Z_{ \pm}^{2}}{d t}=-\alpha \frac{Z_{ \pm}^{2} Z_{\mp}}{\lambda_{ \pm}}
$$

The parameter $\alpha \approx 1$ when the applied magnetic field $B_{0} \approx 0$, and $\alpha \approx \frac{1}{2}$ when $B_{0} \geqslant 1$. This parameter emulates the effects of spectral anisotropy. The model is completed by length-scale equations

$$
\frac{d \lambda_{ \pm}}{d t}=\beta_{ \pm} Z_{\mp}
$$

This model simplifies for $H_{c}=0\left(Z^{2}=Z_{ \pm}^{2}\right)$ to the equivalent of the KármánDryden model, which we adopt as a model for the local nonlinear effect in (4.3),

$$
N_{Z}=\left(\frac{d Z^{2}}{d t}\right)_{\text {nonlinear }}=-\alpha \frac{Z^{3}}{\lambda}
$$

provided that we adopt, as we did in the previous section, the approximation that the correlation lengths are equal for the \pm fields, $\lambda_{ \pm}=\lambda$. This turbulent decay rate, sometimes called the 'Kolmogorov' rate, seems to have been applied for the first time to solar wind heating by Coleman (1968).

We can now assemble the full model for the evolution of turbulent energy that is subject to uniform expansion and nonlinear effects, by writing

$$
\frac{\partial Z^{2}}{\partial t}+U \frac{\partial Z^{2}}{\partial r}+\frac{A U}{r} Z^{2}=-\alpha \frac{Z^{3}}{\lambda}
$$

The parameter $A=1+\frac{1}{3} \sigma_{D}$ for isotropic turbulence, while $A=1+\cos ^{2} \Psi \sigma_{D}$ for 2D turbulence. 
It is easy to transcribe the model length-scale equation by noting that, along with (4.3), (4.12) implies

$$
\frac{\partial \lambda}{\partial t}+U \frac{\partial \lambda}{\partial r}+\frac{(B-A) U}{r} \lambda=N_{\lambda}
$$

where $N_{\lambda}$ is the appropriate nonlinear model term, $A$ is the coefficient appearing in (5.4) and $B$ is the coefficient in (4.12). As we have seen in the previous section, these coefficients vary according to the geometry of the turbulence and the choice of correlation length.

The assembly of the MHD length-scale transport equation is completed by adopting the local nonlinear model suggested by Hossain et al. (1995), which reduces in the present context to

$$
N_{\lambda}=\left(\frac{d \lambda}{d t}\right)_{\text {nonlinear }}=\beta Z .
$$

Once again, this is the MHD analog of the Kármán-Dryden model.

The final form of the length-scale transport equation becomes

$$
\frac{\partial \lambda}{\partial t}+U \frac{\partial \lambda}{\partial r}+\frac{C U}{r} \lambda=\beta Z
$$

where $C=B-A$, and these coefficients are summarized in Table 1 for the various cases we have considered. In the next section we shall always choose $\alpha=1, \beta=\frac{1}{2}$. However, in applications in which the mean field $\mathbf{B}_{0}$ is strong, somwhat smaller values of $\alpha$ may be appropriate, as discussed by Hossain et al. (1995).

\section{Steady Solutions and Discussion}

The equations for MHD transport of non-Alfvénic fluctuations, neglecting terms of order $V_{A} / U,(5.4)$ and (5.7), are formally identical to the hydrodynamic transport equations (2.10) and (2.11), with the exception that the parameters $A$ and $B$ differ. For hydrodynamics, $A$ varies between 1 and 2, while for MHD it ranges from 0 to 2. The hydrodynamic case, not surprisingly, is the $\sigma_{D} \rightarrow 1$ limit of the MHD parameters $A$ and $B$ for all cases considered. Because of this similarity, we shall employ the notation $Z^{2}$ and $u^{2}$ interchangeably.

Here, we consider several solutions to the steady-state transport equations. Note that it is sometimes convenient to write the coefficient $A=1+a$, with $a=\cos ^{2} \Psi \sigma_{D}$ for $2 \mathrm{D}$ turbulence, and $a=\frac{1}{3} \sigma_{D}$ for isotropic turbulence.

The non-turbulent limit of the transport equations, lacking dissipation, can be recovered formally by letting $Z \rightarrow 0$, or equivalently, $\lambda \rightarrow \infty$. A dissipationless solution of interest is the one with $a=0, A=1$, for which the transport equation reduces to

$$
U \frac{d Z^{2}}{d r}+\frac{U}{r} Z^{2}=0
$$

This is equivalent to the familiar WKB transport model (Hollweg 1974; Zhou and Matthaeus 1990b; Verma and Roberts 1993) for non-interacting waves propagating in an expanding wind. Specifying the fluctuating energy density (per unit mass) on a surface $r=r_{0}$, and denoting values at $r=r_{0}$ by a zero subscript, the solution is

$$
Z^{2} / Z_{0}^{2}=r_{0} / r
$$


which corresponds to fluctuation energy per unit volume that behaves as $\rho u^{2} / \rho_{0} u_{0}^{2} \sim$ $\left(r_{0} / r\right)^{3}$, where a density scaling $\rho / \rho_{0}=\left(r_{0} / r\right)^{2}$ is assumed.

When $a=-1$, the 'mixing term' takes on it largest negative value, and $A \rightarrow 0$. In the case of slab or $2 \mathrm{D}$ turbulence, $A=0$ requires that the winding angle $\psi=0$ and that $\sigma_{D}=-1$, corresponding to all the energy in magnetic fluctuations. Thus this case does not occur in hydrodynamics. In the absence of dissipation, the solution is $E_{b}=\left\langle|\mathbf{b}|^{2} / 4 \pi \rho\right\rangle=$ const, or

$$
b / b_{0}=r_{0} / r
$$

where $b$ is the RMS magnetic fluctuation. This is equivalent to the result derived by Jokipii and Kota (1989) for the radial dependence of polar heliospheric magnetic fluctuations.

We turn now to the general case in which turbulent dissipation is present, $A$ is treated as a free parameter, and the transport equation for $\lambda$ enters on equal footing.

For the general case, we find that the correlation length $\lambda$ satisfies

$$
\frac{\lambda}{\lambda_{0}}=\left(\frac{r_{0}}{r}\right)^{A / 2+C} \frac{Z_{0}}{Z} .
$$

It follows that the solutions to (5.4) and (5.7) are simply

$$
\begin{gathered}
\frac{Z^{2}}{Z_{0}^{2}}=\frac{\left(r_{0} / r\right)^{A}}{1+F\left[\left(r / r_{0}\right)^{Q}-1\right]}, \\
\frac{\lambda}{\lambda_{0}}=\left\{1+F\left[\left(r / r_{0}\right)^{Q}-1\right]\right\}^{1 / 2}\left(r / r_{0}\right)^{-C},
\end{gathered}
$$

where the exponents have the values $C=B-A$, and $Q=1+C-\frac{1}{2} A=1+B-\frac{3}{2} A$. The parameters $A$ and $B$ are given in Table 1 . The constant $F=D / Q$, with $D=\left(Z_{0} / U\right) r_{0} / \lambda_{0}$ being the ratio of the expansion time $r_{0} / U$ to the eddy turnover time, $\lambda_{0} / Z_{0}$, at the source.

For large radial distances, the asymptotic forms are easily identified. In general, we see that $Z^{2} \sim r^{-(1+C+A / 2)}$ while $\lambda \sim r^{(1-A / 2-C) / 2}$ as $r \rightarrow \infty$. Several limits are noteworthy. When mixing is absent i.e. $A=1, C=0$, always associated with $\sigma_{D}=0$ in the present model, one finds asymptotic solutions

$$
Z^{2} / Z_{0}^{2} \sim\left(r_{0} / r\right)^{1.5}, \quad \lambda / \lambda\left(r_{0}\right) \sim\left(r / r_{0}\right)^{1 / 4}
$$

This corresponds to equipartition of kinetic and magnetic fluctuations, with energy per unit volume scaling as $r^{-3.5}$, slightly steeper than the WKB scaling (cf. eq. (49) et seq.). In the solar wind this solution may be relevant in undriven regions in the outer heliosphere, because the mixing terms are expected to be weak at large heliocentric distances (Matthaeus et al. 1994; Oughton 1993; Zank et al. 1996). The $r^{1 / 4}$ scaling of the correlation length compares favorably with the observational results of Klein et al. (1991). However, the fluctuation energies in the solar wind near the ecliptic plane do not fall off this steeply (Roberts et al. 1990). It is of some interest that energy scaling similar to $r^{-3.5}$ was reported for some higher latitude Ulysses observations by Forsyth et al. (1996). In addition, this solution corresponds to the asymptotic behaviour of $2 \mathrm{D}$ expanding hydrodynamic turbulence with $\Psi=0$ (i.e. radial field).

The isotropic hydrodynamic limit with radial correlation scale is of general in- 


\section{Phenomenology of turbulence in an expanding medium}

terest. Its solutions scale as

$$
u^{2} / u_{0}^{2} \sim\left(r_{0} / r\right)^{4 / 3} \quad \lambda / \lambda_{0} \sim\left(r / r_{0}\right)^{1 / 3}
$$

as $r / r_{0} \rightarrow \infty$.

The MHD limit in which mixing is strong $(A \rightarrow 0)$ leads to the effective cancellation of the expansion term in (5.4). Formally, the strongest mixing occurs for $2 \mathrm{D}$ turbulence with $\sigma_{D}=-1$ and zero winding angle $\Psi=0$. For an $L_{D}=0$ model, one recovers asymptotically,

$$
Z^{2} / Z_{0}^{2} \sim\left(r_{0} / r\right)^{2}, \quad \lambda / \lambda_{0}=\text { const },
$$

the energy per unit volume scaling as $b^{2} \sim r^{-4}$. This scaling corresponds to Taylor's (1935) solution for homogeneous hydrodynamic turbulence with a constant correlation scale. If instead, for the same kind of turbulence, we model the energy decay as dependent upon the normal correlation scale $L_{N}$, and adopt a closure of the $L_{D}=\sigma_{D} L$ type, the asymptotic behavior changes to

$$
Z^{2} / Z_{0}^{2} \sim\left(r_{0} / r\right), \quad \lambda / \lambda_{0}=\left(r / r_{0}\right)^{1 / 2},
$$

This case corresponds directly to the self-preserving Kármán-Dryden model, with $\alpha=1, \beta=\frac{1}{2}$, in (3.3) and (3.4), and is the slowest radial decay of the turbulence that can occur in the present models. A robust feature of these models is that excess magnetic energy $\left(\sigma_{D}<0\right)$ leads to steeper decay of $Z$ with $r$ (see Table 1), and slower growth of $\lambda$.

\section{Conclusions}

We have investigated a class of simple models for the radial evolution of hydrodynamic and MHD turbulence in a specified radial constant-speed expansion. These models and their solutions provide a simple basis for understanding radial evolution of turbulence in space and astrophysical situations, including solar and stellar winds. We expect that these results will be complementary to more detailed theories for the evolution of turbulence in radial winds (see e.g. Marsch and Tu 1993; Oughton and Matthaeus 1995; Schmidt and Marsch 1995) that may prove to be more accurate, but are far less transparent because of their complexity. Direct application of these ideas to observations of solar wind fluctuations, as well as generalization of the present results to include source terms, is an ongoing effort (Zank et al. 1996).

\section{Acknowledgements}

We thank David Montgomery for his inspiration and leadership in plasma physics and turbulence theory on the occasion of his 60th birthday.

This work was supported in part by NASA Space Physics Theory Program Grant NAGW-2076. W.H.M. is also supported by NSF Grant ATM-9318728. G.P.Z. is also supported in part by NASA Grants NRA-94-SSC-SR\&T-027 and an NSF Young Investigator Award ATM-9357861. S.O. is also supported in part by grants from the Nuffield Foundation (SCI/180/94/400) and the UK PPARC (GR/K98711). 


\section{References}

Barnes, A. 1979 Hydromagnetic waves and turbulence in the solar wind. Solar System Plasma Physics, Vol. I (ed. E. N. Parker, C. F. Kennel and L. J. Lanzerotti), p. 251. NorthHolland, Amsterdam.

Belcher, J.W. and Davis, L. 1971 Large-amplitude Alfvén waves in the interplanetary medium, 2. J. Geophys. Res. 76, 3534.

Bieber, J. W., Wanner, W. and Matthaeus, W. H. 1996 Dominant two-dimensional solar wind turbulence with implications for cosmic ray transport. J. Geophys. Res. 101, 2511. Bradshaw, P., Cebeci, T. and Whitelaw, J. 1981 Engineering Calculation Methods for Turbulent
Flow. Academic Press, New York.

Coleman, P.J. 1968 Turbulence, viscosity, and dissipation in the solar wind plasma. Astrophys. J. 153, 371.

Comte-Bellot, G. and Corrsin, S. 1966 The use of a contraction to improve the isotropy of grid-generated turbulence. J. Fluid Mech. 24, 657.

Dobrowolny, M., Mangeney, A. and Veltri, P. 1980 Fully developed anisotropic hydromagnetic turbulence in interplanetary space. Phys. Rev. Lett. 45, 144.

Dryden, H. L. 1943 A review of the statistical theory of turbulence. Q. Appl. Maths 1, 7.

Elsässer, W. M. 1950 The hydromagnetic equations. Phys. Rev. 79, 183.

Forsyth, R.J., Horbury, T.S., Balogh, A. and Smith, E. J. 1996 Hourly variances of fluctuations in the heliospheric magnetic field out of the ecliptic plane. Geophys. Rev. Lett. 23, 595 .

Goldstein, M. L., R. D. A. and Matthaeus, W. H. 1995 Magnetohydrodynamic turbulence in the solar wind. Ann. Rev. Astron. Astrophys. 33, 283.

Hollweg, J. V. 1974 Transverse Alfvén waves in the solar wind: arbitrary $\mathbf{k}, \mathbf{v}_{0}, \mathbf{B}_{0}$ and $\delta \mathbf{b}$. J. Geophys. Res. 79, 1539.

Hossain, M., Gray, P.C., Pontius, D.H., Matthaeus, W. H. and Oughton, S. 1995 Phenomenology for the decay of energy-containing eddies in homogeneous mhd turbulence. Phys. Fluids 7, 2886.

Jokipii, J. R. and Kota, J. 1989 The polar heliospheric magnetic field. Geophys. Rev. Lett. $16,1$.

Jones, W. P. and Launder, B. 1972 Calculation of low-Reynolds-number phenomena with a 2-equation model of turbulence. Int. J. Heat Mass Transf. 15, 301.

Kármán, T. von and Howarth, L. 1938 On the statistical theory of isotropic turbulence. Proc. R. Soc. Lond. Al64, 192.

Kármán, T. von and Lin, C. C. 1949 On the concept of similarity in the theory of isotropic turbulence. Rev. Mod. Phys. $21,516$.

Kármán, T. von and Lin, C.C. 1987 On the statistical theory of turbulence. Selected Papers of C.C.Lin, Vol. 1, p. 135. World Scientific, Singapore.

Klein, L. W., Roberts, D. A. and Goldstein, M. L. 1991 Anisotropy and minimum variance directions of solar wind fluctuations in the outer heliosphere. J. Geophys. Res. 96, 3779.

Kolmogorov, A. N. 1941 On degeneration of isotropic turbulence in an incompressible viscous liquid. C.R. Acad.Sci. URSS 31, 538.

Kraichnan, R. H. 1965 Inertial-range spectrum of hydromagnetic turbulence. Phys. Fluids 8, 1385.

Marsch, E. and Tu, C.-Y. 1989 Dynamies of correlation functions with Elsässer variables for inhomogeneous MHD turbulence. J. Plasma Phys. 41, 479.

Marsch, E. and Tu, C.-Y. 1993 Modeling results on spatial transport and spectral transfer of solar wind Alfvénic turbulence. J. Geophys. Res. 98, 21045.

Matthaeus, W. H. and Goldstein, M. L. $1982 a$ Measurement of the rugged invariants of magnetohydrodynamic turbulence in the solar wind. J. Geophys. Res. 87, 6011.

Matthaeus, W. H. and Goldstein, M. L. $1982 b$ Stationarity of magnetohydrodynamic fluctuations in the solar wind. J. Geophys. Res. 87, 10347.

Matthaeus, W.H., Goldstein, M.L. and Roberts, D.A. 1990 Evidence for the presence of quasi-two-dimensional nearly incompressible fluctuations in the solar wind. J. Geophys. Res. 95, 20673. 
Matthaeus, W. H., Oughton, S., Pontius, D. and Zhou, Y. 1994 Evolution of energy containing turbulent eddies in the solar wind. J. Geophys. Res. 99, 19267.

Orszag, S.A. 1977 Lectures on the statistical theory of turbulence. Fluid Dynamics. Les Houches Summer School, 1973 (ed. R. Balian and J.-L. Peube), p. 235. Gordon and Breach, New York.

Oughton, S. 1993 Transport of solar wind fluctuations: a turbulence approach. PhD thesis, University of Delaware, Newark.

Oughton, S. and Matthaeus, W.H. 1995 Linear transport of solar wind fluctuations. J. Geophys. Res. 100, 14783.

Roberts, D. A., Goldstein, M. L. and Klein, L. W. 1990 The amplitudes of interplanetary fluctuations: stream structure, heliocentric distance, and frequency dependence. J. Geophys. Res. $95,4203$.

Roberts, D. A., Klein, L. W., Goldstein, M. L. and Matthaeus, W. H. 1987a The nature and evolution of magnetohydrodynamic fluctuations in the solar wind: Voyager observations. J. Geophys. Res. 92, 11021.

Roberts, D. A., Goldstein, M. L., Klein, L. W. and Matthaeus, W. H. $1987 b$ Origin and evolution of fluctuations in the solar wind: Helios observations and Helios-Voyager comparisons. J. Geophys. Res. $92,12023$.

Saffman, P. G. 1967 Note on decay of homogeneous turbulence. Phys. Fluids 10, 1349.

Schmidt, J. M. and Marsch, E. 1995 Spatial transport and spectral transfer of solar wind turbulence composed of Alfvén waves and convective structures. 1: The theoretical model. Ann. Geophys. 13, 459.

Taylor, G. I. 1935 Statistical theory of turbulence. Parts 1-4. Proc. R. Soc. Lond. A151, 421.

Tu, C.-Y. and Marsch, E. 1990 Transfer equations for spectral densities of inhomogeneous mhd turbulence. J. Plasma Phys. 44, 103.

Tu, C.-Y. and Marsch, E. 1993 A model of solar wind fluctuations with two components: Alfvén waves and convective structures. J. Geophys. Res. 98, 1257.

Verma, M.K. and Roberts, D. A. 1993 The radial evolution of the amplitudes of 'dissipationless' turbulent solar wind fluctuations. J. Geophys. Res. 98, 5625.

Verma, M. K., Roberts, D. A., Goldstein, M. L., Ghosh, S. and Stribling, W. T. 1996 A numerical study of the nonlinear cascade of energy in magnetohydrodynamic turbulence. J. Geophys. Res., in press.

Zank, G.P., Matthaeus, W. H. and Smith, C. W. 1996 Evolution of turbulent magnetic fluctuation power with heliocentric distance. J. Geophys. Res. 101, 17093.

Zhou, Y. and Matthaeus, W.H. 1990a Transport and turbulence modeling of solar wind fluctuations. J. Geophys. Res. 95, 10291.

Zhou, Y. and Matthaeus, W. H. $1990 b$ Remarks on transport theories of interplanetary fluctuations. J. Geophys. Res. 95, 14863. 